\title{
Adaptive Control of Space Proximity Missions with Constrained Relative States, Faults and Saturation
}

\author{
Liang Sun ${ }^{\mathrm{a}, \mathrm{b}}$, Jingjing Jiang ${ }^{\mathrm{c}}$ \\ ${ }^{a}$ Key Laboratory of Knowledge Automation for Industrial Processes of Ministry of Education, \\ School of Automation and Electrical Engineering, University of Science and Technology Beijing, Beijing 100086, P.R.China \\ ${ }^{b}$ Institute of Artificial Intelligence, University of Science and Technology Beijing, Beijing 100086, P.R.China \\ ${ }^{c}$ Department of Aeronautical and Automotive Engineering, Loughborough University, Leicestershire, LE11 3TU, United Kingdom
}

\begin{abstract}
This paper studies a relative position and relative orientation control problem of close-range spacecraft proximity missions under control input saturation, actuator faults, relative state constraints, kinematic couplings, parametric uncertainties, and unknown external disturbances. The problem of control input saturation is handled with introducing the outputs of an augmented system into the controller, and relative state constraints are guaranteed by using barrier Lyapunov function in backstepping design. Actuator faults in dynamical model are compensated by element-wise adaptive estimations, while unknown dynamic couplings, parametric uncertainties, and unknown bounded disturbances are compensated by norm-wise adaptive estimations. Based on the developed adaptive nonlinear control strategy, relative motion states uniformly ultimately tend to small adjustable neighborhoods of zero, and if the initial relative states are constrained in the predefined ranges, then relative state constraints will never be violated. Simulation comparison validates the advantages of the control strategy.
\end{abstract}

Keywords: Spacecraft proximity missions, relative motion control, fault-tolerant control, input saturation, relative state constraint.

\section{Nomenclature}

$\mathcal{F}_{i}$

$\mathcal{F}$

$\mathcal{F}_{t}$

O

C

T

$\mathbf{P}$

$\boldsymbol{p}_{t} \in \mathbb{R}^{3}$

$r \in \mathbb{R}^{3}$

$v \in \mathbb{R}^{3}$

$\boldsymbol{r}_{t} \in \mathbb{R}^{3}$

$v_{t} \in \mathbb{R}^{3}$

$\boldsymbol{r}_{e} \in \mathbb{R}^{3}$

$\boldsymbol{v}_{e} \in \mathbb{R}^{3}$

$\boldsymbol{\sigma} \in \mathbb{R}^{3}$

$\omega \in \mathbb{R}^{3}$

$\sigma_{t} \in \mathbb{R}^{3}$

$\omega_{t} \in \mathbb{R}^{3}$

$\sigma_{e} \in \mathbb{R}^{3}$

$\omega_{e} \in \mathbb{R}^{3}$

$m \in \mathbb{R}^{+}$

$m_{t} \in \mathbb{R}^{+}$

$J \in \mathbb{R}^{3 \times 3}$

$J_{t} \in \mathbb{R}^{3 \times 3}$

$\tau \in \mathbb{R}^{3}$

$f \in \mathbb{R}^{3}$
Earth-centered inertial coordinate frame

Chaser's body-fixed coordinate frame

Target's body-fixed coordinate frame

Earth's center of mass

Chaser's center of mass

Target's center of mass

Chaser's desired approaching position

Desired relative position, $\mathrm{m}$

Three-dimensional position of chaser, $\mathrm{m}$

Three-dimensional velocity of chaser, $\mathrm{m} / \mathrm{s}$

Three-dimensional position of target, $\mathrm{m}$

Three-dimensional velocity of target, $\mathrm{m} / \mathrm{s}$

Three-dimensional relative position, $\mathrm{m}$

Three-dimensional relative velocity, $\mathrm{m} / \mathrm{s}$

Attitude parameters of chaser's orientation

Chaser's angular velocity, $\mathrm{rad} / \mathrm{s}$

Attitude parameters of target's orientation

Target's angular velocity, rad/s

Relative attitude parameters

Relative angular velocity, $\mathrm{rad} / \mathrm{s}$

Mass of chaser, $\mathrm{kg}$

Mass of target, $\mathrm{kg}$

Inertial matrix of chaser, $\mathrm{kgm}^{2}$

Inertial matrix of target, $\mathrm{kgm}^{2}$

Control torque input of chaser, $\mathrm{Nm}$

Control force input of chaser, $\mathrm{N}$

$\boldsymbol{w}_{c} \in \mathbb{R}^{3}$
$\boldsymbol{w}_{t} \in \mathbb{R}^{3}$
$\boldsymbol{d}_{c} \in \mathbb{R}^{3}$
$\boldsymbol{d}_{t} \in \mathbb{R}^{3}$
$f_{j, \text { min }} \in \mathbb{R}^{+}$
$f_{j, \text { max }} \in \mathbb{R}^{+}$
$\tau_{j, \text { min }} \in \mathbb{R}^{+}$
$\tau_{j, \text { max }} \in \mathbb{R}^{+}$
$\Gamma_{f} \in \mathbb{R}^{3 \times 3}$
$\Gamma_{\tau} \in \mathbb{R}^{3 \times 3}$
$R \in \mathbb{R}^{3 \times 3}$
$I_{3} \in \mathbb{R}^{3 \times 3}$
$O_{3} \in \mathbb{R}^{3 \times 3}$
$\mu_{g}$
$\|\boldsymbol{a}\|_{1}$
$\|\boldsymbol{a}\|$
$\|A\|$
$\operatorname{tr}\{A\}$
$S(\boldsymbol{x})$

External disturbance torque of chaser, $\mathrm{Nm}$ External disturbance torque of target, $\mathrm{Nm}$ External disturbance force of chaser, $\mathrm{N}$ External disturbance force of target, $\mathrm{N}$ Minimum force output of $j^{\text {th }}$ actuator, $\mathrm{N}$ Maximum force output of $j^{\text {th }}$ actuator, $\mathrm{N}$ Minimum torque output of $j^{\text {th }}$ actuator, $\mathrm{Nm}$ Maximum torque output of $j^{\text {th }}$ actuator, $\mathrm{Nm}$ Effectiveness matrix of force actuators Effectiveness matrix of torque actuators Rotational matrix from $\mathcal{F}_{t}$ to $\mathcal{F}$ Three-dimensional unit matrix Three-dimensional zero matrix Earth's gravitational constant 1-norm for any vector $\boldsymbol{a} \in \mathbb{R}^{n}$ 2-norm for any vector $\boldsymbol{a} \in \mathbb{R}^{n}$ 2-norm for any matrix $A$ Trace of any matrix $A \in \mathbb{R}^{n \times n}$ Skew-symmetric matrix for any $\boldsymbol{x} \in \mathbb{R}^{3}$

\section{Introduction}

Future space missions will require high-precision relative pose control for spacecraft under complicated conditions. Rendezvous and proximity, capture of non-cooperative space target, fueling and servicing in orbit, and large spacecraft construction are the special applications [1]. One of the crucial skill$\mathrm{s}$ is developing the pose tracking controller that can drive the six-degrees-of-freedom (6-DOF) relative motion between two 
spacecraft under the effect of system parameter uncertainties, unknown disturbances, actuator saturation, and actuator faults. Specifically, high-precision controller development should be treated as nonlinear control problems, where relative position tracking and relative attitude synchronization are strongly dynamically coupled with each other [2][3]. To ensure the safety in the relative motion control, the 6-DOF relative velocities should be constrained in the given ranges, so that the risks of undesired high-speed collisions between two spacecraft are avoided in many space programmes.

In the past years, many advanced control schemes have been developed for close-range spacecraft proximity missions. An adaptive passivity control approach was formulated in [4] to achieve asymptotic stability of the relative position motion. For the close proximity of an asteroid, the potential shaping and energy dissipation techniques were employed in the statefeedback control law of relative equilibria of the coupled orbitattitude motion in [5]. A nonlinear model predictive control strategy was developed in [6] for spacecraft proximity missions, where relative velocity constraints were deemed to ensure safety and stability. Then, the tube-based and flatnessbased model predictive controllers were respectively developed in [7] and [8] for spacecraft rendezvous and proximity operations. A robust $H_{\infty}$ adaptive feedback controller was developed to achieve asymptotic pose tracking of chaser in [9]. An adaptive sliding mode control approach was proposed in [10] for spacecraft body-fixed hovering in the proximity of an asteroid, where relative pose dynamics were modeled. Based on the dual-quaternion model of relative pose motion, a non-certainty equivalent adaptive control strategy was proposed in [11]. Recently, a model-dependent adaptive controller was formulated for rendezvous and docking in [12], and the artificial potential field approach was developed for spacecraft rendezvous and proximity missions in [13] and [14] to avoid obstacles.

Generally, actuator output saturation is a vital problem in spacecraft control design, since control input signals are always dependent on the time-varying system states, large initial factors, and model uncertainties [15]. Since the actuator output constraints generally result in mechanical failure and performance deterioration, then the control input saturation problem attracts a lot of attention in many applications. In particular, the spacecraft systems always suffer from the actuator saturation problems, and many approaches have been proposed in recent years. Saturated attitude tracking controllers were proposed in [16] and [17] based on adaptive control and robust control techniques, respectively. A robust gain schedule control approach was developed in [18] for spacecraft relative orbital motion subject to input saturation. Sliding mode control technique was used in [19] to design relative motion control systems for spacecraft rendezvous and proximity missions. Another challenging issue in spacecraft systems is developing a controller to guarantee dynamic performance and steady-state performance subject to actuator failures [20]. Sliding mode control approaches in fault-tolerant control system design are proposed for spacecraft, such as adaptive sliding mode control [21] and time-varying sliding mode control [22]. Although saturated controllers were designed in [16]-[18] and fault tolerant control strategies were designed in [20]-[22] for aerospace systems and other nonlinear systems, few results on saturated fault-tolerant relative orbital and orientation controller was derived for space proximity missions. Therefore, it is very significant to study the saturated fault-tolerant relative motion control approach for space rendezvous and docking systems.

To achieve excellent control performance of space proximity missions, several issues including actuator output saturation, actuator faults, relative state constraints, dynamic couplings, parametric uncertainties, and unknown disturbances cannot be ignored in the relative pose control system design, so that synthesizing control system is more difficult. A saturated adaptive fault-tolerant relative pose controller with considering constrained relative states is developed for space proximity missions in this paper. Besides the issues of input saturation, relative state constraints, dynamic couplings, parametric uncertainties, and unknown disturbances, the actuator faults are simultaneously investigated in space proximity missions. Additionally, comparing with the independent control design for the relative rotation and relative translation in [23], the 6-DOF integrated control design is rigorously developed in this study. Main contributions of the proposed 6-DOF integrated control approach are listed as follows. Firstly, compared with the passive faulttolerant control in [26] and the active fault-tolerant control in [27], the proposed active adaptive fault-tolerant control is independent with the explicit information of actuator faults, while element-wise estimations are introduced to compensate the actuator faults under the relative state constraints. Secondly, the proposed controller with a saturation compensator is capable of simultaneously achieving relative orbital and orientation control without violating actuator physical limitations. Especially, different from the saturation compensator in [28], the proposed anti-windup compensator is more effective and simpler, while the undesired switching mechanism and singularity in [28] are averted in saturation compensator. Thirdly, barrier Lyapunov functions are employed in backstepping technique to ensure the relative state constraints in space proximity missions. Specifically, the barrier Lyapunov function is employed in every step of backstepping procedures rather than only used in the first step as in [29] to ensure the constraints of relative motion states. Fourthly, the uniform boundedness of all signals in closed-loop system is ensured in stability analysis and computer simulation$\mathrm{s}$ are performed for relative pose motion to validate the performance of the control strategy.

The structure of the paper is arranged as follows. The control objective of space proximity missions is described in Section 2. Control design procedures are presented in Section 3 with the rigorously stability analysis. Section 4 gives the simulation results to validate the theoretical results. Section 5 concludes the paper.

\section{Problem Description}

A 6-DOF integrated control strategy is investigated for a controlled chaser tracking an uncontrolled target with unknown disturbances. The models of the chaser and the target are respec- 
tively described in $\mathcal{F}$ and $\mathcal{F}_{t}$ with respect to $\mathcal{F}_{i}$ as [24]

$$
\begin{aligned}
& \left\{\begin{array}{l}
\dot{\boldsymbol{r}}=\boldsymbol{v}-S(\boldsymbol{\omega}) \boldsymbol{r} \\
m \dot{\boldsymbol{v}}+m S(\boldsymbol{\omega}) \boldsymbol{v}+m \mu \boldsymbol{r}=\Gamma_{f} \boldsymbol{f}+\boldsymbol{d}_{c} \\
\dot{\boldsymbol{\sigma}}=G(\boldsymbol{\sigma}) \boldsymbol{\omega} \\
J \dot{\boldsymbol{\omega}}+S(\boldsymbol{\omega}) J \boldsymbol{\omega}=\Gamma_{\tau} \boldsymbol{\tau}+\boldsymbol{w}_{c}
\end{array}\right. \\
& \left\{\begin{array}{l}
\dot{\boldsymbol{r}}_{t}=\boldsymbol{v}_{t}-S\left(\boldsymbol{\omega}_{t}\right) \boldsymbol{r}_{t} \\
m_{t} \dot{\boldsymbol{v}}_{t}+m_{t} S\left(\boldsymbol{\omega}_{t}\right) \boldsymbol{v}_{t}+m_{t} \mu_{t} \boldsymbol{r}_{t}=\boldsymbol{d}_{t} \\
\dot{\boldsymbol{\sigma}}_{t}=G\left(\boldsymbol{\sigma}_{t}\right) \boldsymbol{\omega}_{t} \\
J_{t} \dot{\boldsymbol{\omega}}_{t}+S\left(\boldsymbol{\omega}_{t}\right) J_{t} \boldsymbol{\omega}_{t}=\boldsymbol{w}_{t}
\end{array}\right.
\end{aligned}
$$

where $\sigma$ and $\sigma_{t}$ are modified Rodrigues parameters [30] to describe the attitudes of two spacecraft, and kinematic Jacobian is $G(\boldsymbol{x})=\frac{1}{4}\left[\left(1-\boldsymbol{x}^{\mathrm{T}} \boldsymbol{x}\right) I_{3}+2 S(\boldsymbol{x})+2 \boldsymbol{x} \boldsymbol{x}^{\mathrm{T}}\right]$ for $\boldsymbol{x}=\boldsymbol{\sigma}$ or $\boldsymbol{x}=\boldsymbol{\sigma}_{t}$, respectively; $\Gamma_{\tau}=\operatorname{diag}\left\{\gamma_{\tau 1}, \gamma_{\tau 2}, \gamma_{\tau 3}\right\}$ and $\Gamma_{f}=\operatorname{diag}\left\{\gamma_{f 1}, \gamma_{f 2}, \gamma_{f 3}\right\}$ with $0<\gamma_{i j} \leq 1(i=\tau, f ; j=1,2,3), \gamma_{i j}=1$ means that the $j^{\text {th }}$ actuator is healthy, and $0<\gamma_{i j}<1$ means that the $j^{\text {th }}$ actuator is partially failure.

Let the relative pose and velocities described in $\mathcal{F}$ with respect to $\mathcal{F}_{i}$ be [30]

$$
\left\{\begin{array}{l}
\sigma_{e}=\frac{\sigma_{t}\left(\sigma^{\mathrm{T}} \sigma-1\right)+\sigma\left(1-\sigma_{t}^{\mathrm{T}} \sigma_{t}\right)-2 S\left(\sigma_{t}\right) \sigma}{1+\sigma_{t}^{\mathrm{T}} \sigma_{t} \sigma^{\mathrm{T}} \sigma+2 \sigma_{t}^{\mathrm{T}} \sigma} \\
\boldsymbol{r}_{e}=\boldsymbol{r}-R \boldsymbol{r}_{p_{t}}, \boldsymbol{v}_{e}=\boldsymbol{v}-R \boldsymbol{v}_{p_{t}}, \omega_{e}=\omega-R \omega_{t}
\end{array}\right.
$$

where $\boldsymbol{p}_{t}$ denotes the final position vector of the chaser with respect to the target, $\boldsymbol{r}_{p_{t}}=\boldsymbol{r}_{t}+\boldsymbol{p}_{t}, \boldsymbol{v}_{p_{t}}=\boldsymbol{v}_{t}+S\left(\boldsymbol{\omega}_{t}\right) \boldsymbol{p}_{t}$, and

$$
R=I_{3}-\frac{4\left(1-\sigma_{e}^{\mathrm{T}} \sigma_{e}\right)}{\left(1+\sigma_{e}^{\mathrm{T}} \sigma_{e}\right)^{2}} S\left(\sigma_{e}\right)+\frac{8}{\left(1+\sigma_{e}^{\mathrm{T}} \sigma_{e}\right)^{2}} S^{2}\left(\sigma_{e}\right) .
$$

Furthermore, define 6-DOF system states $\boldsymbol{e}_{1}=\left[\boldsymbol{r}_{e}^{\mathrm{T}}, \boldsymbol{\sigma}_{e}^{\mathrm{T}}\right]^{\mathrm{T}}$ and $\boldsymbol{e}_{2}=\left[\boldsymbol{v}_{e}^{\mathrm{T}}, \omega_{e}^{\mathrm{T}}\right]^{\mathrm{T}}$. The coupled 6-DOF relative dynamics are

$$
\left\{\begin{array}{l}
\dot{\boldsymbol{e}}_{1}=C_{1} \boldsymbol{e}_{1}+C_{2} \boldsymbol{e}_{2} \\
\dot{\boldsymbol{e}}_{2}=\boldsymbol{h}+\boldsymbol{n}_{1}+\boldsymbol{n}_{2}+\Phi \boldsymbol{u}+\boldsymbol{d}
\end{array}\right.
$$

where $C_{1}=\operatorname{diag}\left\{-S(\omega), O_{3}\right\}, C_{2}=\operatorname{diag}\left\{I_{3}, G\left(\sigma_{e}\right)\right\}, \boldsymbol{h}=$ $\left[\begin{array}{c}-\boldsymbol{g} \\ -S(\omega) \omega_{e}\end{array}\right], \boldsymbol{n}_{1}=\left[\begin{array}{c}\mathbf{0} \\ -J^{-1} S(\omega) J \omega\end{array}\right], \boldsymbol{n}_{2}=B J_{t}^{-1} S(\boldsymbol{\eta}) J_{t} \boldsymbol{\eta}$, $\Phi=M^{-1} \Gamma, \boldsymbol{u}=\left[\boldsymbol{f}^{\mathrm{T}}, \boldsymbol{\tau}^{\mathrm{T}}\right]^{\mathrm{T}}, \boldsymbol{d}=\left[\boldsymbol{d}_{1}^{\mathrm{T}}, \boldsymbol{d}_{2}^{\mathrm{T}}\right]^{\mathrm{T}}, \boldsymbol{g}=S(\boldsymbol{\omega}) \boldsymbol{v}_{e}+S^{2}(\boldsymbol{\omega}-$ $\left.\omega_{e}\right) R \boldsymbol{p}_{t}+\mu \boldsymbol{r}-\frac{\mu_{g}\left(\boldsymbol{r}-\boldsymbol{r}_{e}-R \boldsymbol{p}_{t}\right)}{\left\|R^{\mathrm{T}}\left(\boldsymbol{r}-\boldsymbol{r}_{e}\right)-\boldsymbol{p}_{t}\right\|^{3}}, B=\left[\begin{array}{c}-R S\left(\boldsymbol{p}_{t}\right) \\ R\end{array}\right], \boldsymbol{\eta}=R^{\mathrm{T}}\left(\boldsymbol{\omega}-\boldsymbol{\omega}_{e}\right)$, $M=\operatorname{diag}\left\{m I_{3}, J\right\}, \Gamma=\operatorname{diag}\left\{\Gamma_{\tau}, \Gamma_{f}\right\}, \boldsymbol{d}_{1}=\frac{\boldsymbol{d}_{c}}{m}-\frac{R \boldsymbol{d}_{t}}{m_{t}}+R S\left(\boldsymbol{p}_{t}\right) J_{t}^{-1} \boldsymbol{w}_{t}$, $\boldsymbol{d}_{2}=J^{-1} \boldsymbol{w}_{c}-R J_{t}^{-1} \boldsymbol{w}_{t}$.

Suppose that the chaser's control input $\boldsymbol{u}=\left[u_{1}, \cdots, u_{6}\right]^{\mathrm{T}}$ is subject to the following nonsymmetric saturation

$$
-u_{i, \min } \leq u_{i} \leq u_{i, \max }, i=1, \cdots, 6
$$

where $u_{i, \min }=f_{j, \min }$ and $u_{i, \max }=f_{j, \max }$ for $i=1,2,3 ; j=1,2,3$; $u_{i, \min }=\tau_{j, \min }$ and $u_{i, \max }=\tau_{j, \max }$ for $i=4,5,6 ; j=1,2,3$. Thus, the control input $u_{i}$ is defined by

$$
u_{i}= \begin{cases}u_{i, \max } & \text { if } u_{0 i}>u_{i, \max } \\ u_{0 i} & \text { if }-u_{i_{\min }} \leq u_{0 i} \leq u_{i, \max } \\ -u_{i, \min } & \text { if } u_{0 i}<-u_{i, \min }\end{cases}
$$

where $\boldsymbol{u}_{0}=\left[u_{01}, \cdots, u_{06}\right]^{\mathrm{T}}$ denotes the control command to be designed.
Assumption 1. $m, J, m_{t}$, and $J_{t}$ are unknown and constant parameters, where $0<m_{1} \leq m^{-1} \leq m_{2}, 0<\theta_{1} \leq\left\|J^{-1}\right\| \leq \theta_{2}$ with known constants $m_{i}$ and $\theta_{i}(i=1,2) . \quad \boldsymbol{d}_{c}, \boldsymbol{w}_{c}, \boldsymbol{d}_{t}$, and $\boldsymbol{w}_{t}$ are unknown time-varying vectors and bounded by $\left\|\boldsymbol{d}_{c}\right\| \leq$ $\bar{d}_{c},\left\|\boldsymbol{w}_{c}\right\| \leq \bar{w}_{c},\left\|\boldsymbol{d}_{t}\right\| \leq \bar{d}_{t},\left\|\boldsymbol{w}_{t}\right\| \leq \bar{w}_{t}$ with unknown constant bounds $\bar{d}_{c}, \bar{w}_{c}, \bar{d}_{t}$, and $\bar{w}_{t}$. This assumption presents the uncertainties of physical parameters and disturbances.

Assumption 2. In actuator effectiveness matrices $\Gamma_{\tau}$ and $\Gamma_{f}$, the function $\gamma_{i j}$ is unknown but bounded by $0<\gamma_{i j \text {,min }} \leq \gamma_{i j} \leq$ $\gamma_{i j \text {, max }}$, where the constants $\gamma_{i j \text {, min }}$ and $\gamma_{i j \text {,max }}$ are known. This assumption shows that fault information is uncertain.

Assumption 3. The motion variables $\{\boldsymbol{r}, \boldsymbol{v}, \boldsymbol{\sigma}, \boldsymbol{\omega}\}$ and relative motion variables $\left\{\boldsymbol{r}_{e}, \boldsymbol{v}_{e}, \boldsymbol{\sigma}_{e}, \boldsymbol{\omega}_{e}\right\}$ are available by the chaser from the sensors mounted on its body [31][32], but the motion variables $\left\{\boldsymbol{r}_{t}, \boldsymbol{v}_{t}, \boldsymbol{\sigma}_{t}, \boldsymbol{\omega}_{t}\right\}$ cannot be derived directly from the target.

In this work, we aim to design novel control law for space proximity missions subject to actuator saturation and faults, relative state constraints, dynamic couplings, parametric uncertainties, and unknown disturbances. This designing goal is equivalent to synthesizing input command $\boldsymbol{u}_{0}$ with Assumptions 1-3 such that all signals are bounded and relative states are always constrained, namely $\left|e_{1 i}(t)\right|<a_{e i}$ and $\left|e_{2 i}(t)\right|<b_{e i}$ with known constants $a_{e i}$ and $b_{e i}$ for $t \geq 0$.

\section{Control Design and Stability Analysis}

Define $z_{1}=e_{1}$ and $z_{2}=e_{2}-\alpha$ with a virtual input $\alpha$. Then, based on model (3), the $z_{1}$-dynamics can be derived as

$$
\dot{z}_{1}=C_{1} z_{1}+C_{2} z_{2}+C_{2} \alpha
$$

Choose a function

$$
V_{1}=\frac{1}{2} \sum_{i=1}^{6} \log \frac{\boldsymbol{a}_{e}^{\mathrm{T}} E_{i} \boldsymbol{a}_{e}}{\boldsymbol{a}_{e}^{\mathrm{T}} E_{i} \boldsymbol{a}_{e}-z_{1}^{\mathrm{T}} E_{i} z_{1}}
$$

where $\boldsymbol{a}_{e}=\left[a_{e 1}, a_{e 2}, \cdots, a_{e 6}\right]^{\mathrm{T}}$ is the upper bound of $\boldsymbol{e}_{1}$; $E_{1}=\operatorname{diag}\{1,0,0,0,0,0\}, E_{2}=\operatorname{diag}\{0,1,0,0,0,0\}, \cdots, E_{6}=$ $\operatorname{diag}\{0,0,0,0,0,1\}$. Clearly, for $\left|z_{1 i}\right|<a_{e i}(i=1,2, \cdots, 6)$, the Lyapunov function candidate (7) is positive definite and continuous. Then, from (6) and $\sum_{i=1}^{6} z_{1}^{\mathrm{T}} E_{i} C_{1} z_{1}=0$, its time derivative can be derived as

$$
\dot{V}_{1}=\sum_{i=1}^{6} \frac{z_{1}^{\mathrm{T}} E_{i} \dot{z}_{1}}{\boldsymbol{a}_{e}^{\mathrm{T}} E_{i} \boldsymbol{a}_{e}-z_{1}^{\mathrm{T}} E_{i} z_{1}}=\sum_{i=1}^{6} \frac{z_{1}^{\mathrm{T}} E_{i}\left(C_{2} z_{2}+C_{2} \boldsymbol{\alpha}\right)}{\boldsymbol{a}_{e}^{\mathrm{T}} E_{i} \boldsymbol{a}_{e}-z_{1}^{\mathrm{T}} E_{i} z_{1}}
$$

Since $G^{-1}\left(\sigma_{e}\right)=\frac{16}{1+\left\|\sigma_{e}\right\|^{2}} G^{\mathrm{T}}\left(\sigma_{e}\right)$ and $\left\|G\left(\sigma_{e}\right)\right\|=\frac{1+\left\|\sigma_{e}\right\|^{2}}{4}$, then $C_{2}$ is a nonsingular matrix. Design the virtual control

$$
\alpha=-C_{2}^{-1} \Lambda z_{1}
$$

where $\Lambda=\operatorname{diag}\left\{\Lambda_{1}, \Lambda_{2}\right\}$ is a diagonal positive-definite matrix and $\Lambda_{i} \in \mathbb{R}^{3 \times 3}(i=1,2)$. Combining (9) and (8) leads to

$$
\dot{V}_{1}=-\sum_{i=1}^{6} \frac{z_{1}^{\mathrm{T}} E_{i} \Lambda z_{1}}{\boldsymbol{a}_{e}^{\mathrm{T}} E_{i} \boldsymbol{a}_{e}-z_{1}^{\mathrm{T}} E_{i} z_{1}}+\sum_{i=1}^{6} \frac{z_{1}^{\mathrm{T}} E_{i} C_{2} z_{2}}{\boldsymbol{a}_{e}^{\mathrm{T}} E_{i} \boldsymbol{a}_{e}-z_{1}^{\mathrm{T}} E_{i} z_{1}}
$$


Then, the $z_{1}$-dynamics (6) can be rewritten as

$$
\dot{z}_{1}=C_{1} z_{1}+C_{2} z_{2}-\Lambda z_{1}
$$

From the definition of $z_{2}$, we obtain the $z_{2}$-dynamics as

$$
\dot{z}_{2}=\boldsymbol{h}-\dot{\alpha}+n_{1}+n_{2}+\Phi u+d
$$

where $\dot{\alpha}=-\dot{C}_{2}^{-1} \Lambda z_{1}-C_{2}^{-1} \Lambda \dot{z}_{1}, \dot{z}_{1}$ can be calculated from (6), $\dot{C}_{2}^{-1}=\operatorname{diag}\left\{O_{3}, \dot{G}^{-1}\right\}, \dot{G}^{-1}=-G^{-1} \dot{G} G^{-1}, \dot{G}$ is computed by [33]

$$
\begin{aligned}
\dot{G}= & \frac{8}{\left(1+\sigma_{e}^{\mathrm{T}} \sigma_{e}\right)^{2}}\left[\dot{\sigma}_{e} \sigma_{e}^{\mathrm{T}}+\sigma_{e} \dot{\boldsymbol{\sigma}}_{e}^{\mathrm{T}}-\dot{\boldsymbol{\sigma}}_{e}^{\mathrm{T}} \boldsymbol{\sigma}_{e} I_{3}-S\left(\dot{\boldsymbol{\sigma}}_{e}\right)\right] \\
& -\frac{16 \boldsymbol{\sigma}_{e}^{\mathrm{T}} \dot{\boldsymbol{\sigma}}_{e}}{\left(1+\boldsymbol{\sigma}_{e}^{\mathrm{T}} \boldsymbol{\sigma}_{e}\right)^{3}}\left[\left(1-\boldsymbol{\sigma}_{e}^{\mathrm{T}} \boldsymbol{\sigma}_{e}\right) I_{3}-2 S\left(\sigma_{e}\right)+2 \boldsymbol{\sigma}_{e} \boldsymbol{\sigma}_{e}^{\mathrm{T}}\right] .
\end{aligned}
$$

Define unknown parameters $\bar{d}=\frac{\bar{d}_{c}}{m}+\frac{\bar{d}_{t}}{m_{t}}+\bar{w}_{c}\left\|J^{-1}\right\|+$ $\bar{w}_{t}\left\|J_{t}^{-1}\right\|\left(1+\left\|\boldsymbol{p}_{t}\right\|\right), \bar{n}_{1}=\left\|J^{-1}\left|\||| J\|, \bar{n}_{2}=\left\|J_{t}^{-1}\left|\left\|\mid J_{t}\right\|\right.\right.\right.\right.$, and their estimations $\hat{\Phi}, \hat{\bar{d}}, \hat{\bar{n}}_{1}$, and $\hat{\bar{n}}_{2}$. Design the relative pose control command under the conditions $\left|z_{1 i}(0)\right|<a_{e i}$ and $\left|z_{2 i}(0)\right|<b_{z i}(i=$ $1, \cdots, 6)$ as

$$
\begin{aligned}
\boldsymbol{u}_{0}= & \hat{\Phi}^{-1}\left[-\sum_{i=1}^{6} \frac{\boldsymbol{b}_{z}^{\mathrm{T}} E_{i} \boldsymbol{b}_{z}-\boldsymbol{z}_{2}^{\mathrm{T}} E_{i} \boldsymbol{z}_{2}}{\boldsymbol{a}_{e}^{\mathrm{T}} E_{i} \boldsymbol{a}_{e}-\boldsymbol{z}_{1}^{\mathrm{T}} E_{i} z_{1}} C_{2}^{\mathrm{T}} z_{1}-K_{1} z_{2}\right. \\
& +\sum_{i=1}^{6}\left(\boldsymbol{b}_{z}^{\mathrm{T}} E_{i} \boldsymbol{b}_{z}-\boldsymbol{z}_{2}^{\mathrm{T}} E_{i} z_{2}\right) K_{2} \boldsymbol{\zeta}-\boldsymbol{h}+\dot{\boldsymbol{\alpha}} \\
& \left.-\left(\hat{\bar{d}}+\hat{\bar{n}}_{1}\|\boldsymbol{\omega}\|^{2}+\hat{\bar{n}}_{2}\left\|\boldsymbol{\omega}-\boldsymbol{\omega}_{e}\right\|^{2}\right) \operatorname{sign}\left(z_{2}\right)\right]
\end{aligned}
$$

with an augmented linear anti-windup compensator system

$$
\dot{\zeta}=-K_{3} \zeta+u_{\Delta}
$$

and design parameter adaptive laws

$$
\left\{\begin{array}{l}
\dot{\hat{\Phi}}=\operatorname{diag}\left\{\dot{\hat{\phi}}_{1}, \dot{\hat{\phi}}_{2}, \cdots, \dot{\hat{\phi}}_{6}\right\} \\
\dot{\hat{\bar{d}}}=\gamma_{2} \sum_{i=1}^{6} \frac{\left\|z_{2}\right\|_{1}}{\boldsymbol{b}_{z}^{\mathrm{T}} E_{i} \boldsymbol{b}_{z}-\boldsymbol{z}_{2}^{\mathrm{T}} E_{i} z_{2}}-\gamma_{0} \gamma_{2} \hat{\bar{d}} \\
\dot{\hat{\bar{n}}}_{1}=\gamma_{3} \sum_{i=1}^{6} \frac{\|\boldsymbol{\omega}\|^{2}\left\|z_{2}\right\|_{1}}{\boldsymbol{b}_{z}^{\mathrm{T}} E_{i} \boldsymbol{b}_{z}-\boldsymbol{z}_{2}^{\mathrm{T}} E_{i} \boldsymbol{z}_{2}}-\gamma_{0} \gamma_{3} \hat{\bar{n}}_{1} \\
\dot{\hat{\bar{n}}}_{2}=\gamma_{4} \sum_{i=1}^{6} \frac{\left\|\boldsymbol{\omega}-\boldsymbol{\omega}_{e}\right\|^{2}\left\|z_{2}\right\|_{1}}{\boldsymbol{b}_{z}^{\mathrm{T}} E_{i} \boldsymbol{b}_{z}-\boldsymbol{z}_{2}^{\mathrm{T}} E_{i} \boldsymbol{z}_{2}}-\gamma_{0} \gamma_{4} \hat{\bar{n}}_{2}
\end{array}\right.
$$

with

$$
\dot{\hat{\phi}}_{i}= \begin{cases}0 & \text { if } \hat{\phi}_{i}=\phi_{i, \min } \text { and } \xi_{i}<0 \\ \gamma_{1} \xi_{i}-\gamma_{0} \gamma_{1} \hat{\phi}_{i} & \text { if } \hat{\phi}_{i}=\phi_{i, \max } \text { and } \xi_{i}>0\end{cases}
$$

where $\boldsymbol{b}_{z}$ is the constraint of $\boldsymbol{z}_{2} ; K_{i}(i=1,2,3)$ is diagonal positive-definite matrix; $\gamma_{i}(i=0,1,2,3,4)$ and $\delta$ are positive constants; $\zeta=\left[\zeta_{1}, \cdots, \zeta_{6}\right]^{\mathrm{T}}$ denotes the output of the augmented system; $\boldsymbol{u}_{\Delta}=\boldsymbol{u}-\boldsymbol{u}_{0} ; \xi_{i}=\frac{z_{2 i} u_{0 i}}{b_{z i}^{2}-z_{2 i}^{2}}, z_{2 i}, u_{0 i}$, and $b_{z i}$ are elements of vectors $\boldsymbol{z}_{2}, \boldsymbol{u}_{0}$, and $\boldsymbol{b}_{z}$, respectively; $\phi_{i, \text { min }}=$ $\min \left\{m_{1}, \theta_{1}\right\} \times \min \left\{\gamma_{i j, \min }, i=\tau, f ; j=1,2,3\right\}$, and $\phi_{i, \max }=$ $\max \left\{m_{2}, \theta_{2}\right\} \times \max \left\{\gamma_{i j, \max }, i=\tau, f ; j=1,2,3\right\} ; \operatorname{sign}\left(z_{2}\right) \triangleq$ $\left[\operatorname{sign}\left(z_{21}\right), \operatorname{sign}\left(z_{22}\right), \cdots, \operatorname{sign}\left(z_{26}\right)\right]^{\mathrm{T}}$, while $\operatorname{sign}\left(z_{2 i}\right)=0$ if $z_{2 i}=$ $0 ; \operatorname{sign}\left(z_{2 i}\right)=1$ if $z_{2 i}>0 ; \operatorname{sign}\left(z_{2 i}\right)=-1$ if $z_{2 i}<0$.
Choose a function

$$
\begin{aligned}
V_{2}= & V_{1}+\frac{1}{2} \sum_{i=1}^{6} \log \frac{\boldsymbol{b}_{z}^{\mathrm{T}} E_{i} \boldsymbol{b}_{z}}{\boldsymbol{b}_{z}^{\mathrm{T}} E_{i} \boldsymbol{b}_{z}-\boldsymbol{z}_{2}^{\mathrm{T}} E_{i} \boldsymbol{z}_{2}}+\frac{1}{2} \zeta^{\mathrm{T}} \boldsymbol{\zeta} \\
& +\frac{1}{2 \gamma_{1}} \operatorname{tr}\left\{\tilde{\Phi}^{\mathrm{T}} \tilde{\Phi}\right\}+\frac{1}{2 \gamma_{2}} \tilde{\tilde{d}}^{2}+\frac{1}{2 \gamma_{3}} \tilde{\bar{n}}_{1}^{2}+\frac{1}{2 \gamma_{4}} \tilde{\bar{n}}_{2}^{2}
\end{aligned}
$$

where $\tilde{\Phi}=\hat{\Phi}-\Phi, \tilde{\bar{d}}=\hat{\bar{d}}-\bar{d}, \tilde{\bar{n}}_{1}=\hat{\bar{n}}_{1}-\bar{n}_{1}, \tilde{\bar{n}}_{2}=\hat{\bar{n}}_{2}-\bar{n}_{2}$ are estimation errors. Clearly, for $\left|z_{2 i}\right|<b_{z i}(i=1, \cdots, 6), V_{2}$ is a Lyapunov function.

Calculating the time derivative of $V_{2}$ gives rise to

$$
\begin{aligned}
\dot{V}_{2}= & \sum_{i=1}^{6} \frac{\boldsymbol{z}_{1}^{\mathrm{T}} E_{i}\left(C_{2} z_{2}-\Lambda z_{1}\right)}{\boldsymbol{a}_{e}^{\mathrm{T}} E_{i} \boldsymbol{a}_{e}-\boldsymbol{z}_{1}^{\mathrm{T}} E_{i} z_{1}}+\sum_{i=1}^{6} \frac{\boldsymbol{z}_{2}^{\mathrm{T}} E_{i} \dot{z}_{2}}{\boldsymbol{b}_{z}^{\mathrm{T}} E_{i} \boldsymbol{b}_{z}-\boldsymbol{z}_{2}^{\mathrm{T}} E_{i} \boldsymbol{z}_{2}} \\
& +\boldsymbol{\zeta}^{\mathrm{T}} \dot{\boldsymbol{\zeta}}+\frac{1}{\gamma_{1}} \operatorname{tr}\left\{\tilde{\Phi}^{\mathrm{T}} \dot{\hat{\Phi}}\right\}+\frac{1}{\gamma_{2}} \tilde{\tilde{d}} \dot{\bar{d}}+\frac{1}{\gamma_{3}} \tilde{\bar{n}}_{1} \dot{\hat{\bar{n}}}_{1}+\frac{1}{\gamma_{4}} \tilde{\bar{n}}_{2} \dot{\overline{\hat{n}}}_{2}
\end{aligned}
$$

Combining (13), (14) and (18) results in

$$
\begin{aligned}
\dot{V}_{2} \leq & -\sum_{i=1}^{6} \frac{\boldsymbol{z}_{1}^{\mathrm{T}} E_{i} \Lambda z_{1}}{\boldsymbol{a}_{e}^{\mathrm{T}} E_{i} \boldsymbol{a}_{e}-\boldsymbol{z}_{1}^{\mathrm{T}} E_{i} z_{1}}-\sum_{i=1}^{6} \frac{\boldsymbol{z}_{2}^{\mathrm{T}} E_{i} K_{1} z_{2}}{\boldsymbol{b}_{z}^{\mathrm{T}} E_{i} \boldsymbol{b}_{z}-z_{2}^{\mathrm{T}} E_{i} z_{2}} \\
& -\sum_{i=1}^{6} \frac{\boldsymbol{z}_{2}^{\mathrm{T}} E_{i} \tilde{\Phi} \boldsymbol{u}_{0}}{\boldsymbol{b}_{z}^{\mathrm{T}} E_{i} \boldsymbol{b}_{z}-\boldsymbol{z}_{2}^{\mathrm{T}} E_{i} z_{2}}+\sum_{i=1}^{6} \frac{\boldsymbol{z}_{2}^{\mathrm{T}} E_{i} \Phi \boldsymbol{u}_{\Delta}}{\boldsymbol{b}_{z}^{\mathrm{T}} E_{i} \boldsymbol{b}_{z}-\boldsymbol{z}_{2}^{\mathrm{T}} E_{i} z_{2}} \\
& -\sum_{i=1}^{6} \frac{\left(\tilde{\bar{d}}+\tilde{\bar{n}}_{1}\|\boldsymbol{\omega}\|^{2}+\tilde{\bar{n}}_{2}\left\|\boldsymbol{\omega}-\boldsymbol{\omega}_{e}\right\|^{2}\right)\left\|z_{2}\right\|_{1}}{\boldsymbol{b}_{z}^{\mathrm{T}} E_{i} \boldsymbol{b}_{z}-\boldsymbol{z}_{2}^{\mathrm{T}} E_{i} \boldsymbol{z}_{2}} \\
& -\boldsymbol{\zeta}^{\mathrm{T}} K_{3} \boldsymbol{\zeta}+\boldsymbol{\zeta}^{\mathrm{T}} \boldsymbol{u}_{\Delta}+\boldsymbol{z}_{2}^{\mathrm{T}} K_{2} \boldsymbol{\zeta}+\frac{1}{\gamma_{1}} \operatorname{tr}\left\{\tilde{\Phi}^{\mathrm{T}} \dot{\hat{\Phi}\}}\right. \\
& +\frac{1}{\gamma_{2}} \tilde{\bar{d}} \dot{\overline{\hat{d}}}+\frac{1}{\gamma_{3}} \tilde{\bar{n}}_{1} \dot{\overline{\hat{n}}}_{1}+\frac{1}{\gamma_{4}} \tilde{\bar{n}}_{2} \dot{\overline{\hat{n}}}_{2}
\end{aligned}
$$

Since $\boldsymbol{\zeta}^{\mathrm{T}} \boldsymbol{u}_{\Delta} \leq \frac{1}{2}\|\boldsymbol{\zeta}\|^{2}+\frac{1}{2}\left\|\boldsymbol{u}_{\Delta}\right\|^{2}, \boldsymbol{z}_{2}^{\mathrm{T}} E_{i} \tilde{\Phi} \boldsymbol{u}_{0}=\operatorname{tr}\left\{\boldsymbol{u}_{0} \boldsymbol{z}_{2}^{\mathrm{T}} E_{i} \tilde{\Phi}\right\}=$ $\operatorname{tr}\left\{\tilde{\Phi}^{\mathrm{T}} E_{i} \boldsymbol{z}_{2} \boldsymbol{u}_{0}^{\mathrm{T}}\right\}, \frac{\boldsymbol{z}_{2}^{\mathrm{T}} E_{i} \Phi \boldsymbol{u}_{\Delta}}{\boldsymbol{b}_{z}^{\mathrm{T}} E_{i} \boldsymbol{b}_{z}-z_{2}^{\mathrm{T}} E_{i} z_{2}} \leq \frac{\phi_{i, \text { max }}^{2}}{2} \boldsymbol{z}_{2}^{\mathrm{T}} E_{i} \boldsymbol{z}_{2}+\frac{\boldsymbol{u}_{\Delta}^{\mathrm{T}} E_{i} \boldsymbol{u}_{\Delta}}{2\left(\boldsymbol{b}_{z}^{\mathrm{T}} E_{i} \boldsymbol{b}_{z}-z_{2}^{\mathrm{T}} E_{i} z_{2}\right)^{2}}$, and $\boldsymbol{z}_{2}^{\mathrm{T}} E_{i} \boldsymbol{z}_{2} \leq \frac{\left\|\boldsymbol{b}_{z}\right\|^{2} z_{2}^{\mathrm{T}} E_{i} z_{2}}{\boldsymbol{b}_{z}^{\mathrm{T}} E_{i} \boldsymbol{b}_{z}-z_{2}^{\mathrm{T}} E_{i} z_{2}}$, then

$$
\begin{aligned}
& \dot{V}_{2} \leq-\sum_{i=1}^{6} \frac{z_{1}^{\mathrm{T}} E_{i} \Lambda z_{1}}{\boldsymbol{a}_{e}^{\mathrm{T}} E_{i} \boldsymbol{a}_{e}-z_{1}^{\mathrm{T}} E_{i} z_{1}}-\sum_{i=1}^{6} \frac{\boldsymbol{z}_{2}^{\mathrm{T}} E_{i} K_{1} z_{2}}{\boldsymbol{b}_{z}^{\mathrm{T}} E_{i} \boldsymbol{b}_{z}-z_{2}^{\mathrm{T}} E_{i} z_{2}} \\
& +\sum_{i=1}^{6} \frac{\phi_{i, \max }^{2}\left\|\boldsymbol{b}_{z}\right\|^{2} z_{2}^{\mathrm{T}} E_{i} z_{2}}{2\left(\boldsymbol{b}_{z}^{\mathrm{T}} E_{i} \boldsymbol{b}_{z}-z_{2}^{\mathrm{T}} E_{i} z_{2}\right)}+\frac{1}{2}\left\|\boldsymbol{u}_{\Delta}\right\|^{2} \\
& +\sum_{i=1}^{6} \frac{\left\|\boldsymbol{u}_{\Delta}\right\|^{2}}{2\left(\boldsymbol{b}_{z}^{\mathrm{T}} E_{i} \boldsymbol{b}_{z}-z_{2}^{\mathrm{T}} E_{i} z_{2}\right)^{2}}+z_{2}^{\mathrm{T}} K_{2} \zeta \\
& -\zeta^{\mathrm{T}}\left(K_{3}-\frac{1}{2} I_{6}\right) \zeta-\sum_{i=1}^{6} \frac{\operatorname{tr}\left\{\tilde{\Phi}^{\mathrm{T}} E_{i} z_{2} \boldsymbol{u}_{0}^{\mathrm{T}}\right\}}{\boldsymbol{b}_{z}^{\mathrm{T}} E_{i} \boldsymbol{b}_{z}-\boldsymbol{z}_{2}^{\mathrm{T}} E_{i} z_{2}} \\
& -\sum_{i=1}^{6} \frac{\left(\tilde{\bar{d}}+\tilde{\bar{n}}_{1}\|\boldsymbol{\omega}\|^{2}+\tilde{\bar{n}}_{2}\left\|\boldsymbol{\omega}-\boldsymbol{\omega}_{e}\right\|^{2}\right)\left\|z_{2}\right\|_{1}}{\boldsymbol{b}_{z}^{\mathrm{T}} E_{i} \boldsymbol{b}_{z}-\boldsymbol{z}_{2}^{\mathrm{T}} E_{i} z_{2}} \\
& +\frac{1}{\gamma_{1}} \operatorname{tr}\left\{\tilde{\Phi}^{\mathrm{T}} \dot{\hat{\Phi}}\right\}+\frac{1}{\gamma_{2}} \tilde{\bar{d}} \dot{\hat{\bar{d}}}+\frac{1}{\gamma_{3}} \tilde{\bar{n}}_{1} \dot{\overline{\bar{n}}}_{1}+\frac{1}{\gamma_{4}} \tilde{\bar{n}}_{2} \dot{\overline{\hat{n}}}_{2}
\end{aligned}
$$

When $\dot{\hat{\phi}}_{i}=0, \frac{1}{\gamma_{1}} \operatorname{tr}\left\{\tilde{\Phi}^{\mathrm{T}} \dot{\hat{\Phi}}\right\}=0$, and

$$
-\sum_{i=1}^{6} \frac{\operatorname{tr}\left\{\tilde{\Phi}^{\mathrm{T}} E_{i} z_{2} \boldsymbol{u}_{0}^{\mathrm{T}}\right\}}{\boldsymbol{b}_{z}^{\mathrm{T}} E_{i} \boldsymbol{b}_{z}-\boldsymbol{z}_{2}^{\mathrm{T}} E_{i} \boldsymbol{z}_{2}}=-\sum_{i=1}^{6} \frac{\tilde{\phi}_{i} z_{2 i} u_{0 i}}{b_{z i}^{2}-z_{2 i}^{2}}=-\sum_{i=1}^{6} \tilde{\phi}_{i} \xi_{i},
$$


if $\hat{\phi}_{i}=\phi_{i, \min }$ and $\xi_{i}<0$, then $\tilde{\phi}_{i}=\hat{\phi}_{i}-\phi_{i} \leq 0$ and

$$
-\sum_{i=1}^{6} \frac{\operatorname{tr}\left\{\tilde{\Phi}^{\mathrm{T}} E_{i} z_{2} \boldsymbol{u}_{0}^{\mathrm{T}}\right\}}{\boldsymbol{b}_{z}^{\mathrm{T}} E_{i} \boldsymbol{b}_{z}-z_{2}^{\mathrm{T}} E_{i} z_{2}} \leq 0
$$

if $\hat{\phi}_{i}=\phi_{i, \max }$ and $\xi_{i} \geq 0$, then $\tilde{\phi}_{i}=\hat{\phi}_{i}-\phi_{i} \geq 0$ and

$$
-\sum_{i=1}^{6} \frac{\operatorname{tr}\left\{\tilde{\Phi}^{\mathrm{T}} E_{i} z_{2} \boldsymbol{u}_{0}^{\mathrm{T}}\right\}}{\boldsymbol{b}_{z}^{\mathrm{T}} E_{i} \boldsymbol{b}_{z}-z_{2}^{\mathrm{T}} E_{i} z_{2}} \leq 0
$$

When $\dot{\hat{\phi}}_{i} \neq 0$, then

$$
-\sum_{i=1}^{6} \frac{\operatorname{tr}\left\{\tilde{\Phi}^{\mathrm{T}} E_{i} z_{2} \boldsymbol{u}_{0}^{\mathrm{T}}\right\}}{\boldsymbol{b}_{z}^{\mathrm{T}} E_{i} \boldsymbol{b}_{z}-z_{2}^{\mathrm{T}} E_{i} z_{2}}+\frac{1}{\gamma_{1}} \operatorname{tr}\left\{\tilde{\Phi}^{\mathrm{T}} \dot{\hat{\Phi}}\right\}=-\gamma_{0} \operatorname{tr}\left\{\tilde{\Phi}^{\mathrm{T}} \hat{\Phi}\right\} .
$$

Thus, it is always holds that

$$
-\sum_{i=1}^{6} \frac{\operatorname{tr}\left\{\tilde{\Phi}^{\mathrm{T}} E_{i} z_{2} \boldsymbol{u}_{0}^{\mathrm{T}}\right\}}{\boldsymbol{b}_{z}^{\mathrm{T}} E_{i} \boldsymbol{b}_{z}-z_{2}^{\mathrm{T}} E_{i} z_{2}}+\frac{1}{\gamma_{1}} \operatorname{tr}\left\{\tilde{\Phi}^{\mathrm{T}} \dot{\hat{\Phi}}\right\} \leq-\gamma_{0} \operatorname{tr}\left\{\tilde{\Phi}^{\mathrm{T}} \hat{\Phi}\right\} .
$$

Furthermore, we know

$$
\begin{gathered}
\operatorname{tr}\left\{\tilde{\Phi}^{\mathrm{T}} \hat{\Phi}\right\}=\operatorname{tr}\left\{\tilde{\Phi}^{\mathrm{T}}(\tilde{\Phi}+\Phi)\right\}=\frac{1}{2}\|\tilde{\Phi}\|_{F}^{2}-\frac{1}{2}\|\Phi\|_{F}^{2}+\frac{1}{2}\|\tilde{\Phi}+\Phi\|_{F}^{2} \\
\tilde{\boldsymbol{\vartheta}}^{\mathrm{T}} \hat{\boldsymbol{\vartheta}}=\tilde{\boldsymbol{\vartheta}}^{\mathrm{T}}(\tilde{\boldsymbol{\vartheta}}+\boldsymbol{\vartheta})=\frac{1}{2}\|\tilde{\boldsymbol{\vartheta}}\|^{2}-\frac{1}{2}\|\boldsymbol{\vartheta}\|^{2}+\frac{1}{2}\|\tilde{\boldsymbol{\vartheta}}+\boldsymbol{\vartheta}\|^{2}
\end{gathered}
$$

where $\|A\|_{F}$ denotes the Frobenius-norm of any $A \in \mathbb{R}^{n \times n}, \boldsymbol{\vartheta}=$ $\left[\bar{d}, \bar{n}_{1}, \bar{n}_{2}\right]^{\mathrm{T}}$. Moreover, from [29], we also know

$$
\begin{aligned}
& \log \frac{a_{e i}^{2}}{a_{e i}^{2}-z_{1 i}^{2}} \leq \frac{z_{1 i}^{2}}{a_{e i}^{2}-z_{1 i}^{2}}, z_{1 i} \in\left\{z_{1 i} \in \mathbb{R} \| z_{1 i} \mid<a_{e i}, a_{e i}>0\right\}, \\
& \log \frac{b_{z i}^{2}}{b_{z i}^{2}-z_{2 i}^{2}} \leq \frac{z_{2 i}^{2}}{b_{z i}^{2}-z_{2 i}^{2}}, z_{2 i} \in\left\{z_{2 i} \in \mathbb{R} \| z_{2 i} \mid<b_{z i}, b_{z i}>0\right\} .
\end{aligned}
$$

Substitute (15) into (20) and choose controller parameters such that $\beta_{1}>0, \beta_{2}>\left\|\boldsymbol{b}_{z}\right\|^{2} \beta_{3}^{2}+\frac{1}{2}\left\|\boldsymbol{b}_{z}\right\|^{2} \sum_{i=1}^{6} \phi_{i, \max }^{2}$, and $\beta_{4}>1$, where $\beta_{i}(i=1,2,3,4)$ are minimum eigenvalues of gain matrices $\Lambda$, $K_{1}, K_{2}$, and $K_{3}$, respectively. Then we have

$$
\begin{aligned}
\dot{V}_{2} \leq & -\sum_{i=1}^{6} \frac{\beta_{1}\left\|z_{1}\right\|^{2}}{\boldsymbol{a}_{e}^{\mathrm{T}} E_{i} \boldsymbol{a}_{e}-\boldsymbol{z}_{1}^{\mathrm{T}} E_{i} z_{1}}-\sum_{i=1}^{6} \frac{\beta_{z}\left\|z_{2}\right\|^{2}}{\boldsymbol{b}_{z}^{\mathrm{T}} E_{i} \boldsymbol{b}_{z}-z_{2}^{\mathrm{T}} E_{i} \boldsymbol{z}_{2}} \\
& -\left(\beta_{4}-1\right)\|\boldsymbol{\zeta}\|^{2}-\gamma_{0}\left(\operatorname{tr}\left\{\tilde{\Phi}^{\mathrm{T}} \hat{\Phi}\right\}+\tilde{\bar{d}} \hat{\bar{d}}+\tilde{\bar{n}}_{1} \hat{\bar{n}}_{1}\right. \\
& \left.+\tilde{\bar{n}}_{2} \hat{\bar{n}}_{2}\right)+\frac{1}{2}\left\|\boldsymbol{u}_{\Delta}\right\|^{2}+\sum_{i=1}^{6} \frac{\left\|\boldsymbol{u}_{\Delta}\right\|^{2}}{2\left(\boldsymbol{b}_{z}^{\mathrm{T}} E_{i} \boldsymbol{b}_{z}-z_{2}^{\mathrm{T}} E_{i} \boldsymbol{z}_{2}\right)^{2}} \\
\leq & -\sum_{i=1}^{6} \frac{\beta_{1}\left\|z_{1}\right\|^{2}}{\boldsymbol{a}_{e}^{\mathrm{T}} E_{i} \boldsymbol{a}_{e}-\boldsymbol{z}_{1}^{\mathrm{T}} E_{i} z_{1}}-\sum_{i=1}^{6} \frac{\beta_{z}\left\|z_{2}\right\|^{2}}{\boldsymbol{b}_{z}^{\mathrm{T}} E_{i} \boldsymbol{b}_{z}-\boldsymbol{z}_{2}^{\mathrm{T}} E_{i} \boldsymbol{z}_{2}} \\
& -\left(\beta_{4}-1\right)\|\boldsymbol{\zeta}\|^{2}-\frac{\gamma_{0}}{2}\left(\|\tilde{\Phi}\|_{F}^{2}+\|\tilde{\boldsymbol{\vartheta}}\|^{2}\right)+s \\
\leq & -\mu V_{2}+s
\end{aligned}
$$

where $\beta_{z}=\beta_{2}-\left\|\boldsymbol{b}_{z}\right\|^{2} \beta_{3}^{2}-\frac{1}{2}\left\|\boldsymbol{b}_{z}\right\|^{2} \sum_{i=1}^{6} \phi_{i, \max }^{2}, \mu=$ $\min \left\{2 \beta_{1}, 2 \beta_{z}, 2\left(\beta_{4}-1\right), \gamma_{1} / \gamma_{0}, \gamma_{2} / \gamma_{0}, \gamma_{3} / \gamma_{0}, \gamma_{4} / \gamma_{0}\right\}$ and $s=$ $\frac{\gamma_{0}}{2}\|\Phi\|_{F}^{2}+\frac{\gamma_{0}}{2}\|\boldsymbol{\vartheta}\|^{2}+\frac{1}{2}\left\|\boldsymbol{u}_{\Delta}\right\|^{2}+\sum_{i=1}^{6} \frac{\left\|\boldsymbol{u}_{\Delta}\right\|^{2}}{2\left(\boldsymbol{b}_{z}^{\mathrm{T}} E_{i} \boldsymbol{b}_{z}-z_{2}^{\mathrm{T}} E_{i} z_{2}\right)^{2}}$.
Theorem 1. Given the relative pose model of space proximity missions (3) under Assumptions 1-3 and a mild assumption that $\left\|\boldsymbol{u}_{\Delta}\right\| \leq \bar{u}_{\Delta}$ with an unknown constant $\bar{u}_{\Delta}>0$. If initial relative states are constrained by $\left|e_{1 i}(0)\right|<a_{e i}$ and $\left|e_{2 i}(0)\right|<b_{e i}(i=1, \cdots, 6)$, and the saturated adaptive controller (13) is designed with an auxiliary system (14) and adaptive laws in (15) under parameter tuning conditions $\beta_{1}>0$, $\beta_{2}>\left\|\boldsymbol{b}_{z}\right\|^{2} \beta_{3}^{2}+\frac{1}{2}\left\|\boldsymbol{b}_{z}\right\|^{2} \sum_{i=1}^{6} \phi_{i, \max }^{2}$, and $\beta_{4}>1$, then the relative motion states $\boldsymbol{e}_{1}$ and $\boldsymbol{e}_{2}$ ultimately tend to small neighborhood$s$ of origin by tuning appropriate parameters, while the constraints of relative states are never violated, namely $\left|e_{1 i}(t)\right|<a_{e i}$ and $\left|e_{2 i}(t)\right|<b_{e i}$ with positive constants $a_{e i}$ and $b_{e i}$ for all $t \geq 0$.

Proof: Integrating the time derivative of $V_{2}$ in (21) yields

$$
0<V_{2}(t) \leq\left[V_{2}(0)-\frac{\bar{s}}{\mu}\right] \mathrm{e}^{-\mu t}+\frac{\bar{s}}{\mu}
$$

where

$$
\bar{s}=\frac{\gamma_{0}}{2}\|\Phi\|_{F}^{2}+\frac{\gamma_{0}}{2}\|\boldsymbol{\vartheta}\|^{2}+\frac{1}{2} \bar{u}_{\Delta}^{2}+\sum_{i=1}^{6} \frac{\bar{u}_{\Delta}^{2}}{2\left(\boldsymbol{b}_{z}^{\mathrm{T}} E_{i} \boldsymbol{b}_{z}-z_{2}^{\mathrm{T}} E_{i} z_{2}\right)^{2}} .
$$

This means that $\left\|z_{1}\right\|,\left\|z_{2}\right\|,\|\zeta\|,\|\tilde{\Phi}\|_{F}$, and $\|\tilde{\boldsymbol{\vartheta}}\|$ are uniformly bounded. Specifically, larger $\mu$ results in smaller ultimate bounds of system states. Then invoking (17) and (22) yields

$$
\begin{aligned}
& \frac{1}{2} \log \frac{a_{e i}^{2}}{a_{e i}^{2}-z_{1 i}^{2}} \leq \frac{1}{2} \sum_{i=1}^{6} \frac{a_{e i}^{2}}{a_{e i}^{2}-z_{1 i}^{2}} \leq V_{2}(t), \\
& \frac{1}{2} \log \frac{b_{z i}^{2}}{b_{z i}^{2}-z_{2 i}^{2}} \leq \frac{1}{2} \sum_{i=1}^{6} \frac{b_{z i}^{2}}{b_{z i}^{2}-z_{2 i}^{2}} \leq V_{2}(t) .
\end{aligned}
$$

Taking exponentials on both sides of (23) and (24) yields

$$
\left\{\begin{array}{l}
\left|z_{1 i}(t)\right| \leq a_{e i} \sqrt{1-\mathrm{e}^{-2\left[\left(V_{2}(0)-\bar{s} / \mu\right) \mathrm{e}^{-\mu t}+\bar{s} / \mu\right]}}<a_{e i} \\
\left|z_{2 i}(t)\right| \leq b_{z i} \sqrt{1-\mathrm{e}^{-2\left[\left(V_{2}(0)-\bar{s} / \mu\right) \mathrm{e}^{-\mu t}+\bar{s} / \mu\right]}}<b_{z i}
\end{array}\right.
$$

with $i=1, \cdots, 6$. Since $G^{-1}\left(\sigma_{e}\right)=\frac{16}{1+\left\|\sigma_{e}\right\|^{2}} G^{\mathrm{T}}\left(\sigma_{e}\right),\left\|G\left(\sigma_{e}\right)\right\|=$ $\frac{1+\left\|\sigma_{e}\right\|^{2}}{4}$, then $\left\|C_{2}^{-1}\right\| \leq 1+\left\|G^{-1}\right\| \leq 5$. Thus, from $e_{1}=z_{1}$ and $\boldsymbol{e}_{2}=z_{2}+\alpha=z_{2}-C_{2}^{-1} \Lambda z_{1}$, we know

$$
\begin{gathered}
\left|e_{2 i}(t)\right|<a_{e i}, \\
\left|e_{2 i}(t)\right| \leq\left|z_{2 i}(t)\right|+5 \beta_{1}\left|z_{1 i}\right|<b_{z i}+5 \beta_{1} a_{e i} \triangleq b_{e i} .
\end{gathered}
$$

Clearly, if the initial relative states satisfy $\left|e_{1 i}(0)\right|<a_{e i}$ and $\left|e_{2 i}(0)\right|<b_{e i}$, then the constraints of relative states are never violated, namely $\left|e_{1 i}(t)\right|<a_{e i}$ and $\left|e_{2 i}(t)\right|<b_{e i}$ for all $t \geq 0$.

Remark 1. It is noted that the state of the anti-windup compensator (14) is $\zeta$, while this state is employed to deal with actuator saturation effects in (13). The feature of this approach is that the error signal $\boldsymbol{u}_{\Delta}$ between actuator output $\boldsymbol{u}$ and the control command $\boldsymbol{u}_{0}$ can be measured and fed back via the time-varying negative feedback gain $K_{2}$ in (13), so that the control signal and system states can be indirectly affected by this compensator [23]. The anti-windup compensator (14) is a bounded input bounded output linear systems with negative gain. Moreover, 
$\zeta(t)$ is the ultimately uniformly bounded signal from Theorem 1 . Thus, the error signal $\boldsymbol{u}_{\Delta}$ always keeps bounded, and the size of small neighborhood of system states could be regulated by setting appropriate designing parameters.

Remark 2. The response rate will be faster and control precision will be higher under larger $\Lambda$, but larger $\Lambda$ results in larger undesired noises so that the control precision will be decreased [23]. Furthermore, larger $K_{1}$ will also enlarge the undesired noises in the manifold $z_{2}$. The conditions $\beta_{1}>0$, $\beta_{2}>\left\|\boldsymbol{b}_{z}\right\|^{2} \beta_{3}^{2}+\frac{1}{2}\left\|\boldsymbol{b}_{z}\right\|^{2} \sum_{i=1}^{6} \phi_{i, \max }^{2}$, and $\beta_{4}>1$ should be satisfied to guarantee the stability of the anti-windup compensator and the overall system. The adaption bandwidth of the estimated parameters is critically determined by $\gamma_{i}(i=1,2,3,4)$.

Remark 3. The controller (13) uses the discontinuous signum function such that the control chattering phenomenon may be excited. Thus, the boundary layer technique in the traditional variable structure control can be employed to overcome this problem. Specifically, the saturation function $\operatorname{sat}\left(z_{2}\right)=$ $\left[\operatorname{sat}\left(z_{21}\right), \operatorname{sat}\left(z_{22}\right), \cdots, \operatorname{sat}\left(z_{26}\right)\right]^{T}$ is employed to interchange the signum function $\operatorname{sign}\left(z_{2}\right)$ in $(13)$, and the saturation function $\operatorname{sat}\left(z_{2}\right)$ is defined by

$$
\operatorname{sat}\left(z_{2}\right)=\left\{\begin{array}{cc}
\operatorname{sign}\left(z_{2}\right) & \left\|z_{2}\right\|>\delta \\
\frac{1}{\delta} z_{2} & \left\|z_{2}\right\| \leq \delta
\end{array}\right.
$$

where $\delta>0$ is a small constant. Then, using the modified controller, the closed-loop stability could also be proved based on the similar method as the proof of Theorem 1.

\section{Numerical Simulations}

The effectiveness and advantages of the developed controller is validated in this simulation example. Thus, a scenario of autonomous chaser approaching to a space target in the close range is simulated. All parameters of two spacecraft are [24]

$$
\begin{gathered}
m=58.2(\mathrm{~kg}), \quad m_{t}=5425.6(\mathrm{~kg}), \\
J=\left[\begin{array}{ccc}
38 & -2.5 & -5.5 \\
-2.5 & 44 & -2.7 \\
-5.5 & -2.7 & 36
\end{array}\right]\left(\mathrm{kgm}^{2}\right), \\
J_{t}=\left[\begin{array}{ccc}
3336 & -135.4 & -154.2 \\
-135.4 & 3184 & -148.5 \\
-154.2 & -148.5 & 2423
\end{array}\right]\left(\mathrm{kgm}^{2}\right) .
\end{gathered}
$$

The disturbances on two spacecraft are assumed to be

$$
\begin{aligned}
& \boldsymbol{d}_{c}=\left[\begin{array}{l}
3-4 \cos \left(\omega_{o} t\right)-2 \sin \left(\omega_{o} t\right) \\
3-2 \cos \left(\omega_{o} t\right)+3 \sin \left(\omega_{o} t\right) \\
2+2 \cos \left(\omega_{o} t\right)+4 \sin \left(\omega_{o} t\right)
\end{array}\right] \times 10^{-4}+\chi_{1}(\mathrm{~N}), \\
& \boldsymbol{w}_{c}=\left[\begin{array}{l}
6-5 \cos \left(\omega_{o} t\right)+4 \sin \left(\omega_{o} t\right) \\
7+4 \cos \left(\omega_{o} t\right)-5 \sin \left(\omega_{o} t\right) \\
4-3 \cos \left(\omega_{o} t\right)+3 \sin \left(\omega_{o} t\right)
\end{array}\right] \times 10^{-5}+\chi_{2}(\mathrm{Nm}),
\end{aligned}
$$

$\boldsymbol{d}_{t}=100 \boldsymbol{d}_{c}$, and $\boldsymbol{w}_{t}=100 \boldsymbol{w}_{c}$, where $\chi_{1}=0.002 \times \operatorname{randn}(3,1)$, $\chi_{2}=0.0002 \times \operatorname{randn}(3,1), \operatorname{randn}(3,1)$ is the random noise; $\omega_{o}=$ $\sqrt{\mu_{g} /\|\boldsymbol{r}\|^{3}}$ and $\mu_{g}=3.986 \times 10^{14}\left(\mathrm{~m}^{3} / \mathrm{s}^{2}\right)$. Actuator effectiveness matrices are assumed to be

$$
\begin{gathered}
\Gamma_{\tau}=\left[\begin{array}{ccc}
0.5+0.3 \mathrm{e}^{-0.4 t} & 0 & 0 \\
0 & 0.4\left(1+\mathrm{e}^{-0.5 t}\right) & 0 \\
0 & 0 & 0.6+0.2 \mathrm{e}^{-0.3 t}
\end{array}\right]+0.15 \sin \left(\frac{\pi}{6} t\right) I_{3} \\
\Gamma_{f}=\left[\begin{array}{ccc}
0.4\left(1+\mathrm{e}^{-0.3 t}\right) & 0 & 0 \\
0 & 0.7+0.1 \mathrm{e}^{-0.5 t} & 0 \\
0 & 0 & 0.4\left(1+\mathrm{e}^{-0.4 t}\right)
\end{array}\right]+0.18 \sin \left(\frac{\pi}{9} t\right) I_{3}
\end{gathered}
$$

Initial motion states for the chaser and initial relative motion states are all set in Table 1, and the related designing parameters for simulations are presented in Table 2.

\begin{tabular}{ccc}
\multicolumn{3}{c}{ Table 1: States information for the relative motion system } \\
\hline State & Value & Unit \\
\hline $\boldsymbol{r}(0)$ & {$[1,1,1]^{\mathrm{T}} \times 7.078 \times 10^{6}$} & $\mathrm{~m}$ \\
$\boldsymbol{v}(0)$ & {$[2,3,-2]^{\mathrm{T}}$} & $\mathrm{m} / \mathrm{s}$ \\
$\boldsymbol{\sigma}(0)$ & {$[0,0,0]^{\mathrm{T}}$} & - \\
$\omega(0)$ & {$[0,0,0]^{\mathrm{T}}$} & $\mathrm{rad} / \mathrm{s}$ \\
$\boldsymbol{r}_{e}(0)$ & {$[50 / \sqrt{2}, 0,-50 / \sqrt{2}]^{\mathrm{T}}$} & $\mathrm{m}$ \\
$\boldsymbol{v}_{e}(0)$ & {$[0.5,-0.5,0.5]^{\mathrm{T}}$} & $\mathrm{m} / \mathrm{s}$ \\
$\boldsymbol{\sigma}_{e}(0)$ & {$[0.5,-0.6,0.7]^{\mathrm{T}}$} & - \\
$\omega_{e}(0)$ & {$[0.02,-0.02,0.02]^{\mathrm{T}}$} & $\mathrm{rad} / \mathrm{s}$ \\
$\boldsymbol{p}_{t}$ & {$[0,5,0]^{\mathrm{T}}$} & $\mathrm{m}$ \\
\hline
\end{tabular}

Table 2: The related parameters in relative motion simulation

\begin{tabular}{cccc}
\hline Parameter & Value & Parameter & Value \\
\hline$\Lambda$ & $0.04 I_{6}$ & $K_{1}$ & $\left(0.12 I_{3}, 20 I_{3}\right)$ \\
$K_{2}$ & $0.005 I_{6}$ & $K_{3}$ & $1.2 I_{6}$ \\
$\lambda$ & 0.5 & $\delta$ & 0.05 \\
$m_{1}$ & 0.01 & $m_{2}$ & 0.02 \\
$\theta_{1}$ & 0.03 & $\theta_{2}$ & 0.04 \\
$\hat{\phi}_{i}(0)$ & 0.1 & $\hat{\bar{d}}(0)$ & 0 \\
$\hat{\bar{n}}_{1}(0)$ & 0.1 & $\hat{\bar{n}}_{2}(0)$ & 0 \\
$\gamma_{0}$ & 0.002 & $\gamma_{i}$ & 0.02 \\
$\gamma_{\tau i \text { min }}$ & 0.2 & $\gamma_{f i, \min }$ & 0.2 \\
$\gamma_{\tau i, \max }$ & 1 & $\gamma_{f i, \max }$ & 1 \\
$f_{i, \min }$ & 10 & $f_{i, \max }$ & 20 \\
$\tau_{i, \min }$ & 1 & $\tau_{i, \max }$ & 2 \\
$\phi_{i, \min }$ & 0.002 & $\phi_{i, \max }$ & 0.04 \\
$a_{e i}(i=1,2,3)$ & 50 & $a_{e j}(j=4,5,6)$ & 1 \\
$b_{e i}(i=1,2,3)$ & 1.5 & $b_{e j}(j=4,5,6)$ & 0.03 \\
$\zeta_{i}(0)(i=1,2,3)$ & 10 & $\zeta_{j}(0)(j=4,5,6)$ & 2 \\
\hline
\end{tabular}

The three-dimensional position trajectories of two spacecraft in space are shown in Fig.1, and the time histories of system states are presented in Figs.2-6 based on the developed control law (13). In Fig.2, relative position and relative velocity converge to small neighborhoods of zero in $150(\mathrm{~s})$, and they are always within the constraints in the dynamic response. The convergence of relative position and velocity means that two 
spacecraft have no relative orbital motion. In Fig.3, the relative orientation and its angular velocity also converge to small neighborhoods of zero in 200(s), and they are also always constrained in the dynamic response. The convergence of relative orientation and relative angular velocity means that the attitude motions of two spacecraft have been synchronized. The results in Figs.2-3 perform the satisfied relative position tracking and attitude synchronization for space proximity missions. In Fig.4, the actuator outputs are always constrained in the prescribed range, and the adverse effect of control saturation is handled. From Figs.5-6, we know that the adaptive estimations are always bounded, and the state signals of the augmented system$\mathrm{s}$ also ultimately tend to small neighborhoods of origin. In a word, Figs.2-6 imply that the ultimately uniform stability of space proximity control system is achieved, while the developed control law could achieve reliable and safe space proximity missions with saturated control inputs.

For sake of showing the merits of the developed control law in this work, Figs.7-9 present some simulation results based on the adaptive backstepping control law in [23], where input saturation and relative states constraints are taken into account in the relative motion control design. From Figs.7-9, the relative orbital and relative orientation asymptotically converge to small neighborhoods of origin, but the transient response time is overlong, although the constraints of relative states are never violated obviously during the transient response. Comparing Figs.7-9 with Figs.2-4, the dynamic response time based on the developed control law is clearly smaller than the ones based on the control law in [23]. In spite of the easy designing of adaptive backstepping controller in [23], it is different to guarantee good dynamic performance with small convergent time for space proximity missions.

To quantitatively describe the advantages of the develope$\mathrm{d}$ control approach, there are three indexes to evaluate the dynamic and steady-state response performances and control effort of two control laws, such as the integrated time absolute error (ITAE), the integrated absolute error (IAE), and the integral of square error (ISE), and defined by ITAE = $\sum_{i=1}^{6} \int_{0}^{t} \tau_{s}\left|z_{1 i}\left(\tau_{s}\right)\right| \mathrm{d} \tau_{s}, I A E=\sum_{i=1}^{6} \int_{0}^{t}\left|z_{1 i}\left(\tau_{s}\right)\right| \mathrm{d} \tau_{s}$, and ISE $=$ $\sum_{i=1}^{6} \int_{0}^{t} u_{i}^{2}\left(\tau_{s}\right) \mathrm{d} \tau_{s}$, where $t$ is set as the whole simulation time 400 (s). For the developed adaptive control law (13), the three indexes are

$$
I T A E_{1}=70660.3417, I A E_{1}=253.4708, I S E_{1}=502.8436 .
$$

Meanwhile, for the controller in [23], these three indexes are

$$
I T A E_{2}=81193.6631, I A E_{2}=283.6204, I S E_{2}=577.5773 .
$$

Comparing these three indexes between two controllers, it is known that the developed adaptive control strategy has preferable performance with respect to the control law in [23] with

$$
I T A E_{1}<I T A E_{2}, I A E_{1}<I A E_{2}, I S E_{1}<I S E_{2}
$$

under the same conditions of actuator saturation, modeling uncertainties, and relative state constraints. Therefore, the proposed controller can complete the safe and reliable proximity missions with satisfied control performance.

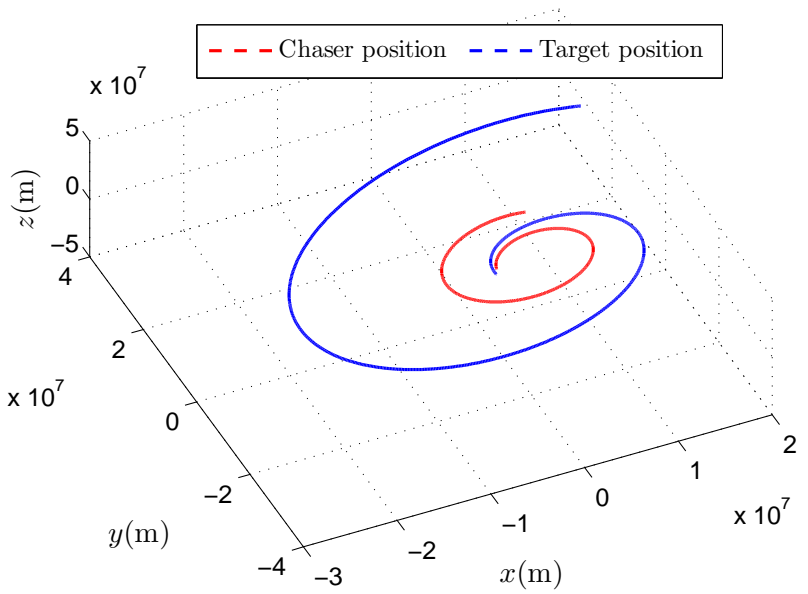

Figure 1: Three-dimensional position trajectory with control law (13).
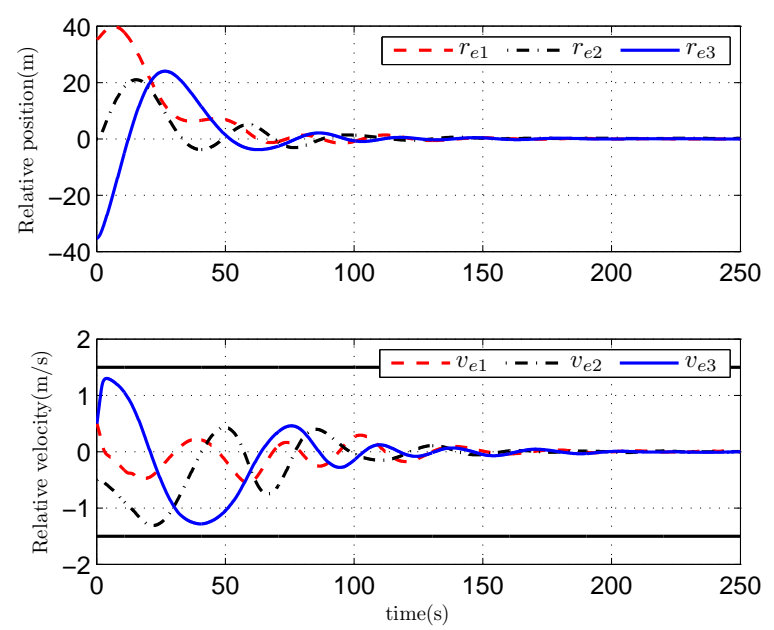

Figure 2: Relative position and velocity with control law (13).
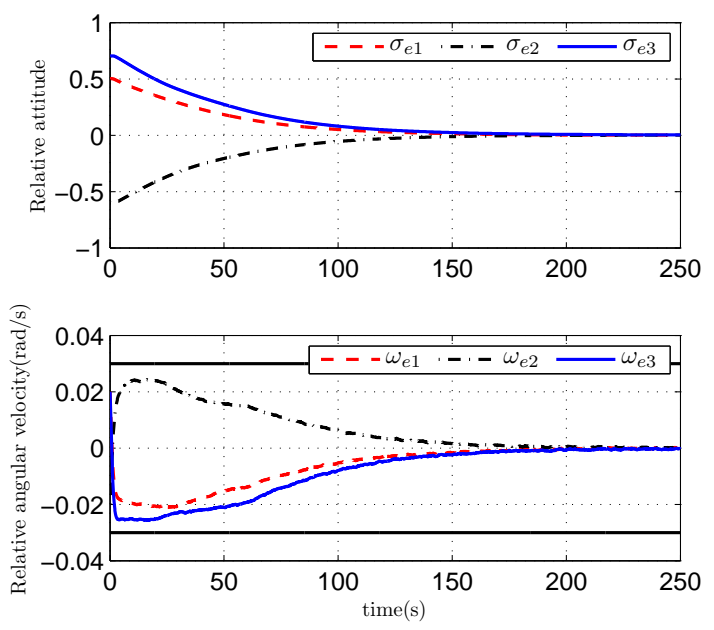

Figure 3: Relative attitude and angular velocity with control law (13). 

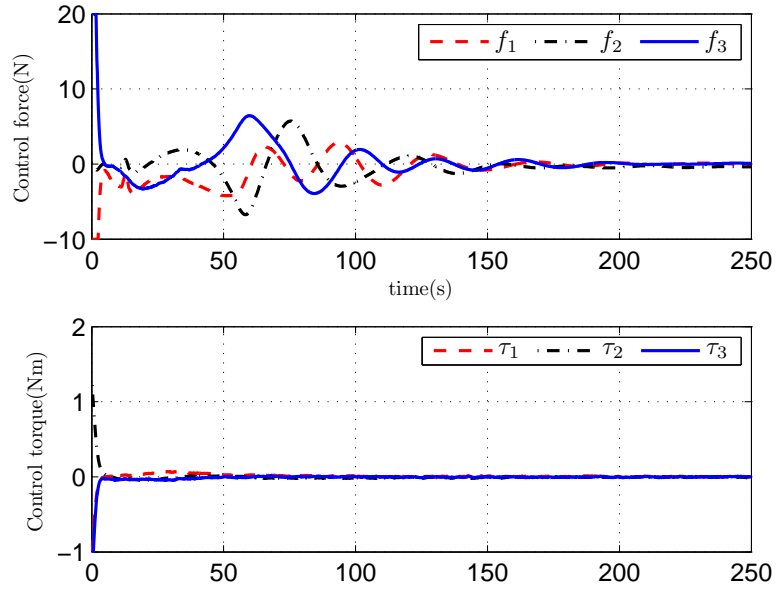

Figure 4: Force and torque inputs with control law (13).

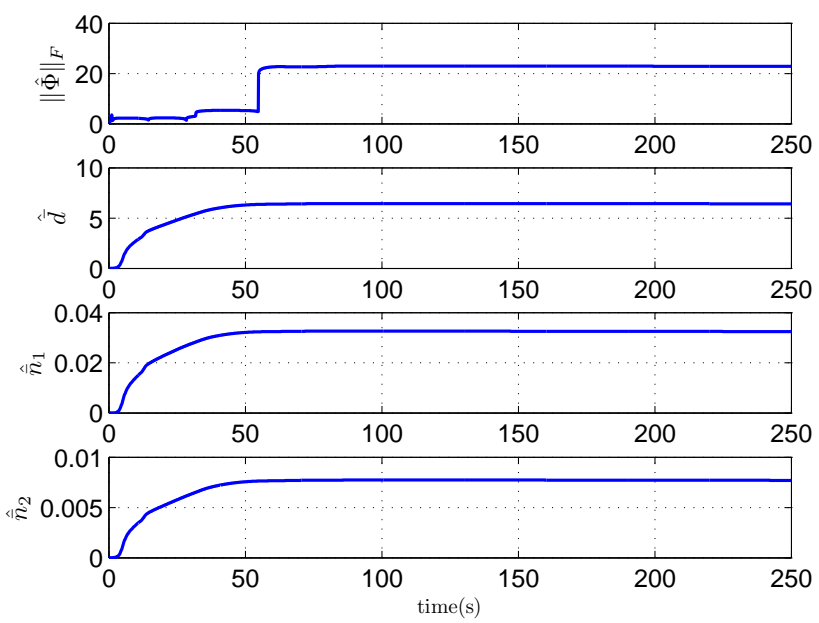

Figure 5: Parameter estimations with adaptive law (15).
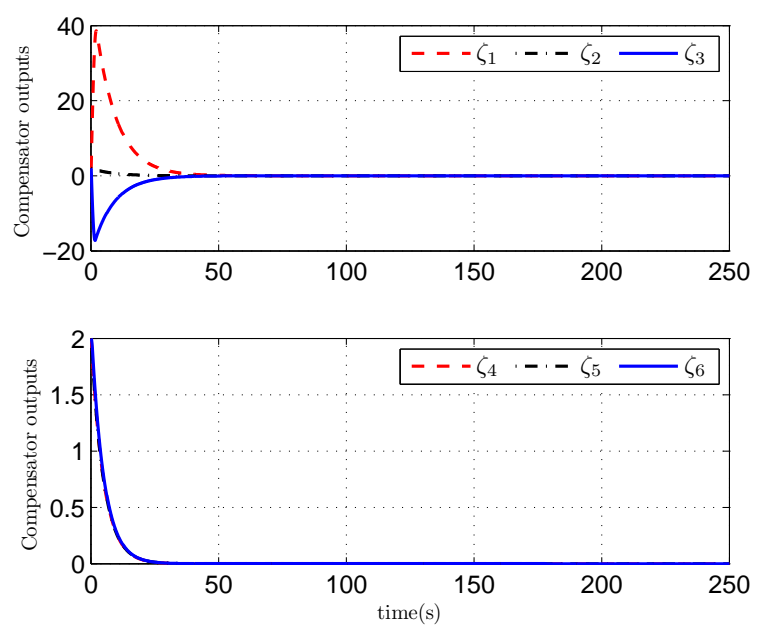

Figure 6: Outputs of augmented system (14).
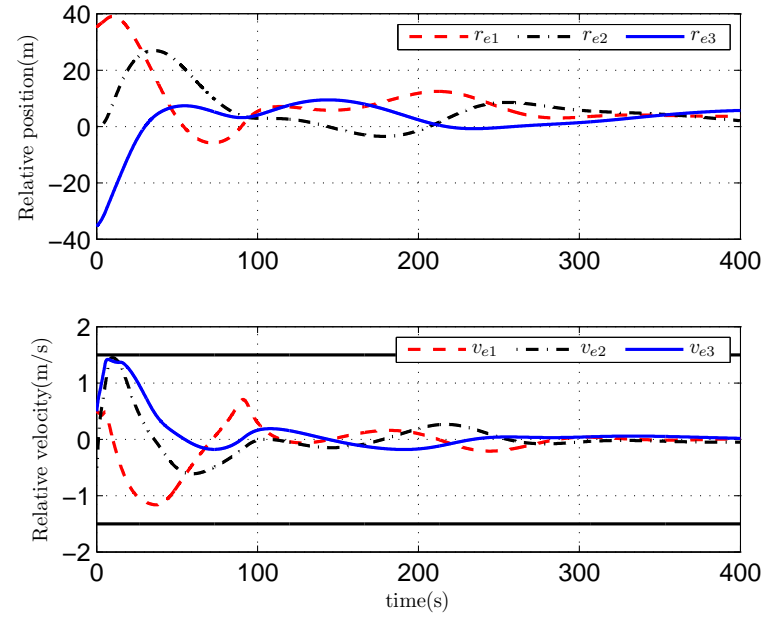

Figure 7: Relative position and velocity with control law in [23].
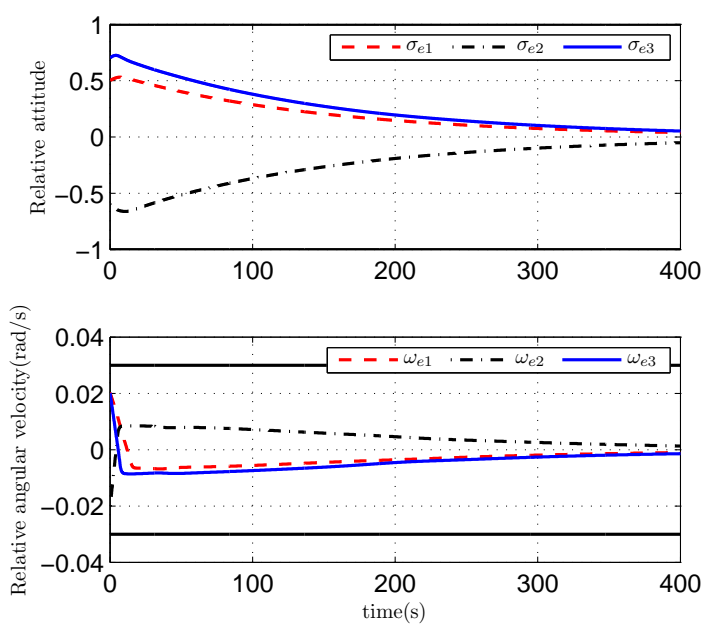

Figure 8: Relative attitude and angular velocity with control law in [23].
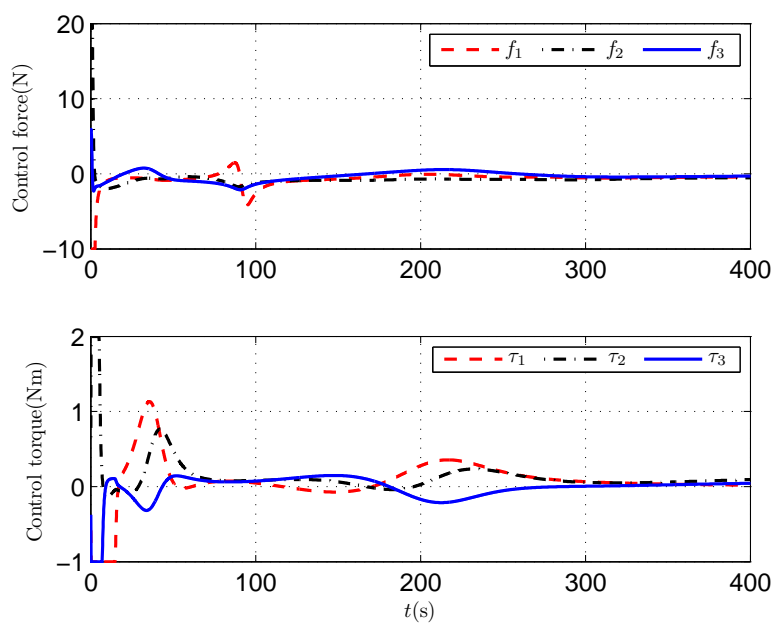

Figure 9: Force and torque inputs with control law in [23]. 


\section{Conclusions}

An adaptive nonlinear control approach is developed for synthesizing the relative pose controller of autonomous space proximity missions under actuator saturation, actuator faults, relative state constraints, dynamic couplings, parametric uncertainties, and unknown disturbances. Given the designed adaptive laws and augmented system, the six-degrees-of-freedom integrated relative pose control law could ensure that all relative states tend to small neighborhood of origin, while control input saturation and relative state constraints are never violated during spacecraft rendezvous and proximity operations. Simulation results demonstrate the performance of the developed control approach. The theoretical analysis and simulation example indicate that the developed control strategy can complete the satisfied autonomous control of close-range proximity missions under multiple complicated conditions. Moreover, the developed control method in this paper can be easily extended and applied to other similar nonlinear systems.

\section{Acknowledgment}

This work was supported by the National Natural Science Foundation of China [grant number 61903025], the China Scholarship Council [grant number 201906465028], and the Fundamental Research Funds for the Central Universities [grant number FRF-BD-19-002A].

\section{References}

[1] J. L. Goodman, "History of space shuttle rendezvous and proximity operations," J. Spacecraft Rockets, vol. 43, no. 5, pp. 944-959, 2006.

[2] P.Z. Schulte and D. A. Spencer, "Development of an integrated spacecraft guidance, navigation and control subsystem for automated proximity operations," Acta Astronaut., vol. 118, pp. 168-186, 2016.

[3] M. Wilde, C. Clark, and M. Romano, "Historical survey of kinematic and dynamic spacecraft simulators for laboratory experimentation of on-orbit proximity maneuvers," Prog. Aerosp. Sci., vol. 110, no.100552, 2019.

[4] S. Ulrich, A. Saenz-Otero, and I. Barkana, "Passivity-based adaptive control of robotic spacecraft for proximity operations under uncertainties," $J$. Guid. Control Dyn., vol. 39, no. 6, pp. 1441-1450, 2016.

[5] Y. Wang, and S. Xu, "Stabilization of coupled orbit-attitude dynamics about an asteroid utilizing Hamiltonian structure," Astrodynamics, vol. 2, no. 1, pp. 53-67, 2018.

[6] S. Di Cairano, H. Park, and I. Kolmanovsky, "Model predictive control approach for guidance of spacecraft rendezvous and proximity maneuvering," Int. J. Robust Nonlinear Control, vol. 22, no. 12, pp. 1398-1427, 2012.

[7] M. Mammarella, E. Capello, H. Park, G. Guglieri, M. Romano, "Tubebased robust model predictive control for spacecraft proximity operations in the presence of persistent disturbance," Aerosp. Sci. Technol., vol. 77, pp. 585-594, 2018.

[8] J. C. Sanchez, F. Gavilan, R. Vazquez, and C. Louembet, "A flatnessbased predictive controller for six-degrees of freedom spacecraft rendezvous," Acta Astronaut., vol. 167, pp. 391-403, 2020.

[9] Y. Ikeda, T. Kida, and T. Nagashio, "Nonlinear tracking control of rigid spacecraft under disturbance using PID-type $H_{\infty}$ adaptive state feedback," Trans. Japan Society Aeronaut. Space Sci., vol. 58, no. 5, pp. 289-297, 2015.

[10] D. Lee and G. Vukovich, "Adaptive sliding mode control for spacecraft body-fixed hovering in the proximity of an asteroid," Aerosp. Sci. Technol., vol.46, pp. 471-483, 2015.
[11] D. Seo, "Fast adaptive pose tracking control for satellites via dual quaternion upon non-certainty equivalence principle," Acta Astronaut., vol. 115, pp. 32-39, 2015.

[12] G. Vukovich and H. Gui, "Robust adaptive tracking of rigid-body motion with applications to asteroid proximity operations," IEEE Trans. Aerosp. Electron. Syst., vol. 53, no. 1, pp. 419-430, 2017.

[13] R. Zappulla, H. Park, J. Virgili-Llop, and M. Romano,"Real-time autonomous spacecraft proximity maneuvers and docking using an adaptive artificial potential field approach," IEEE Trans. Control Syst. Technol., vol. 27, no. 6, pp. 2598-2605, 2019.

[14] M. Mancini, N. Bloise, E. Capello, and E. Punta, "Sliding mode control techniques and artificial potential field for dynamic collision avoidance in rendezvous maneuvers," IEEE Control Syst. Lett., vol. 4, no. 2, pp. 313-318, 2020.

[15] N. Fischer, A. Dani, N. Sharma, and W. E. Dixon, "Saturated control of an uncertain nonlinear system with input delay," Automatica, vol. 49, pp. 1741-1747, 2013.

[16] A. H. J. De Ruiter, "Adaptive spacecraft attitude tracking control with actuator saturation," J. Guid. Control Dyn., vol. 33, no. 5, pp. 1692-1696, 2010 .

[17] A. Zou, K. D. Kumar, and A. H. J. De Ruiter, "Robust attitude tracking control of spacecraft under control input magnitude and rate saturation," Int. J. Robust Nonlinear Control, vol. 26, no. 4, pp. 799-815, 2016.

[18] Q. Wang, B. Zhou, and G. Duan, "Robust gain schedule control of spacecraft rendezvous system subject to input saturation," Aerosp. Sci. Technol., vol. 42, pp. 442-450, 2015.

[19] S. A. Kasaeian, M. Ebrahimi, and N. Assadian, "Closed-loop poweredcoast-powered predictive guidance for spacecraft rendezvous with nonsingular terminal sliding mode steering," Acta Astronaut., vol. 166, pp. 507-523, 2020.

[20] L. Sun, "Adaptive fault-tolerant constrained control of cooperative spacecraft rendezvous and docking," IEEE Trans. Ind. Electron., vol. 67, no. 4, pp. 3107-3115, 2020.

[21] S. Varma and K. D. Kumar, "Fault tolerant satellite attitude control using solar radiation pressure based on nonlinear adaptive sliding mode," Acta Astronaut., vol. 66, no. 3/4, pp. 486-500, 2010.

[22] Q. Hu, X. Shao, and W.-H. Chen, "Robust fault-tolerant tracking control for spacecraft proximity operations using time-varying sliding mode," IEEE Trans. Aerosp. Electron. Syst., vol. 54, no. 1, pp. 2-17, Feb. 2018.

[23] L. Sun, W. Huo, and Z. Jiao, "Adaptive backstepping control of spacecraft rendezvous and proximity operations with input saturation and full-state constraint," IEEE Trans. Ind. Electron., vol. 64, no. 1, pp. 480-492, 2017.

[24] L. Sun, W. Huo and Z. Jiao, "Disturbance observer-based robust relative pose control for spacecraft rendezvous and proximity operations under input saturation," IEEE Trans. Aerosp. Electron. Syst., vol. 54, no. 4, pp.1605-1617, 2018.

[25] W. Guan and G. Yang, "Adaptive fault-tolerant control of linear systems with actuator saturation and disturbances," J. Control Theory Appl., vol. 7, no. 2, pp. 119-126, 2009.

[26] A.-M. Zou and K. D. Kumar, "Adaptive fuzzy fault-tolerant attitude control of spacecraft," Control Eng. Pract., vol. 19, no. 1, pp. 10-21, 2011.

[27] K. Xia and W. Huo, "Adaptive control for spacecraft rendezvous subject to actuator faults and saturations," ISA Trans., vol. 80, pp. 176-186, 2018.

[28] M. Chen, S. S. Ge, and B. Ren. "Adaptive tracking control of unceratin MIMO nonlinear systems with input constraints," Automatica, vol. 47, no. 3, pp. 452-465, Mar. 2011.

[29] K. P. Tee and S. S. Ge, "Control of nonlinear systems with partial state constraints using a barrier Lyapunov function," Int. J. Control, vol. 84, no. 12, pp. 2008-2023, 2011.

[30] H. Schaub and J. L. Junkins, Analytical Mechanics of Space Systems, Reston: AIAA, 2003.

[31] R. Opromolla, G. Fasano, G. Rufino, and M. Grassi, "A review of cooperative and uncooperative spacecraft pose determination techniques for close-proximity operations," Prog. Aerosp. Sci., vol. 93, pp. 53-72, 2017.

[32] G. Lentaris, I. Stratakos, I. Stamoulias, D. Soudris, M. Lourakis, and X. Zabulis, "High-performance vision-based navigation on soc FPGA for spacecraft proximity operations," IEEE Trans. Circu. Syst. Video Technol., in press, avaliable online, doi: 10.1109/TCSVT.2019.2900802.

[33] A. H. J. De Ruiter and C. J. Damaren, Effect of attitude parameterization on the perfermance of passivity-based adaptive attitude control. AIAA Guid. Nav. Control Conf. Exih., 2001, AIAA-2001-4154. 